\title{
Entropic corrections to Friedmann equations in the extra dimensional FRW universe
}

\begin{abstract}
In this work, the quantum gravitational effects on the dynamical equations of universe have been investigated by utilizing the generalized uncertainty principle (GUP) and modified dispersion relation (MDR). At first, the corrected entropy-area relation has been obtained by considering the impacts of GUP and MDR, separately. Next, making use of the corrected entropy relations, arisen from GUP and MDR approaches, the entropic corrected Friedmann equations have been obtained in the extra dimensional FRW universe. The corrections are worked out up to the sixth order of the Planck length. Finally, it has been shown that these two alternative approaches lead to the same corrections if the expansion coefficients satisfy some simple conditions.
\end{abstract}

Volume 2 Issue 6 - 2018

\author{
M Dehghani \\ Department of physics, Razi University, Kermanshah, Iran \\ Correspondence: M Dehghani, Department of physics, Razi \\ University, Kermanshah, Iran, Tel 098-9183888637, \\ Email dehghen22@gmail.com
}

Received: August 20, 2018| Published: December 12, 2018

Keywords: generalized uncertainty principle, modified dispersion relation, Friedmann equations, Friedmann-Robertson-Walker universe

\section{Introduction}

There are several motivations for studying thermodynamical properties of spacetimes more than four dimensions. The first comes from string theory which contains gravity and requires more than four dimensions. In superstring theory a ten-dimensional spacetime is necessary to be consistent from the quantum point of view. The other originates from AdS/CFT correspondence. It relates properties of a $d$-dimensional spacetimes to those of a conformal field theory in (d-1)dimensions. In addition, it is possible for the higher dimensional black holes to be produced in the future colliders based on the large extra dimensions and Tev-scale gravity scenarios. ${ }^{1,2}$ We believe that study of higher dimensional space times in the presence of quantum gravity effects (GUP and MDR) can essentially lead to a deeper insight into under- standing of the ultimate quantum gravity proposals as well as the physical properties of the higher dimensional space times.

Ted Jacobson was the first one who studied the connections between gravity and thermo-dynamics. He showed that the gravitational Einstein equation can be derived from the entropy relation as a function of the horizon area and together with the Clausius relation. ${ }^{3}$ The discovery of relation between quantum tunnelling radiation and black hole entropy, as a pure geometrical quantity, further supports this connection and the thermodynamic interpretation of geometrical

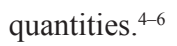

The connection between gravity and thermodynamics has been investigated in various gravity theories..$^{7-12}$ Now it is believed that one is able to derive Friedmann equations by use of the Clausius relation to the apparent horizon of FRW universe, in which entropy is assumed to be proportional to its horizon area.

Making use of the holographic principle together with the equipartition theorem and the Unruh temperature, Cai et al derived the Friedmann equations in FRW universe. ${ }^{13}$ The modified FRW Friedmann equations have been obtained by applying different entropyarea relations together with the first law of thermodynamics..$^{14,15}$

Furthermore, it is commonly believed that one of the most important achievements in perturbative string theory and quantum gravity is the prediction of a fundamental measurable length which is of the order of Planck length. The essence of this fundamental length can be captured by generalizing usual uncertainty principle known as GUP or by modifying the usual energy momentum relation conventionally named as MDR. ${ }^{16-22}$

Recently, the GUP and MDR has been the subject of many interesting works and a lot of papers have appeared in which the usual uncertainty principle is generalized at the framework of microphysics. ${ }^{23-30}$ The GUP corrections to the entropy of black holes have been obtained by several authors based on the Cardy-Verlinde formula. ${ }^{31-33}$ The influence of Gup on the Friedmann equations has been investigated in $(\mathrm{n}+1)$-dimensional FRW universe by Zhu et al. ${ }^{34}$. Also the modification of energy-momentum relations and its applications have been investigated extensively. ${ }^{35-38}$ Furthermore, the extra dimensional version of the MDR has been proposed by direct comparison with the extra dimensional form of GUP..$^{39}$ The proposed extra dimensional MDR has been applied to obtain the first order corrections to the entropy of d-dimensional Schwarzschild black hole through the Cardy-Verlinde formula. ${ }^{33}$ To study the entropic correction to Friedmann equations in the higher dimensional FRW universe, it is interesting to relate the entropy of the universe with a minimal length quantum gravity scale.

Modification of Friedmann equations have been studied by several authors using different approaches. For example, Liu et al. ${ }^{40}$ have obtained correction terms to the first Friedmann equation in four dimensional FRW universe, starting from the holographic principle and Debye model by treating the gravity as an entropic force (according to the Verlinve's proposal). ${ }^{40}$ Also Sheykhi et al..$^{41-43}$ have obtained the same corrections to the first Friedmann equation in four dimensional FRW universe, making use of the Verlinve proposal and applying the quantum gravity corrected entropy-area relation. Sheykhi et al. ${ }^{44}$ by treating the gravity as an entropic force, have obtained correction terms to the same Friedmann equation arising from power-law corrected entropy-area relation. Cai et al. ${ }^{45,46}$ have obtained correction terms to the first and second FRW Friedmann equations, making use of alternative entropy-area relations, in the Einstein, Gauss-Bonnet and Lovelock gravity theories. 
In this work, motivated by characterizing the primordial inflation, we would like to obtain the quantum corrected Friedmann equations from a more fundamental starting point and by taking into account the higher order correction terms. For these purposes, we calculate the GUP and MDR corrections to the Friedmann equations for any spacial curvature in the extra dimensional FRW universe. The corrections are calculated separately in the framework of (1) the generalized uncertainty principle and (2) the modified dispersion relation. The results of these alternative approaches are compared and it is shown that the results are compatible if the constant coefficients are fixed properly. We believe that it can essentially lead to a deeper insight into the ultimate quantum gravity proposals.

The organization of this paper is based on the following order In section-2, the $(\mathrm{n}+1)$-dimensional FRW universe and Friedmann equations are reviewed briefly and the basic equations are introduced. Section-3 is devoted to obtain the entropic corrections to the Friedmann equations in the framework of GUP. In section-4 the corrections from modified entropy-area relation to the Friedmann equations are worked out making use of the MDR analysis. The corrections are obtained up to the sixth power of the Planck length. In section-5, we compare the results of these two alternative approaches and show that a suitable choice of the expansion coefficients leads to the same corrections to the Friedmann equations in the $(\mathrm{n}+1)$-dimensional FRW universe. The results are summarized and discussed in section-6.2 Review of $(n+1)$ dimensional FRW universe and Friedmann equations.

\section{Review of $(n+I)$-dimensional FRW universe and Friedmann equations}

Let's begin with the $d=(n+1)$-dimensional FRW universe, whose line element is described by ${ }^{34}$

$$
d s^{2}=-d t^{2}+a^{2}\left(\frac{d r^{2}}{1-k r^{2}}+r^{2} d \Omega_{n-1}\right)=h_{a b} d x^{a} d x^{b}+\tilde{\mathbf{r}}^{2} d \Omega_{n-1},
$$

where $\Omega_{n-1}$ is the line element of an (n-1)-dimensional unit sphere, $x^{0}=t, x^{1}=r, h_{a b}=\operatorname{diag}\left(-1, a^{2} /\left(1-k r^{2}\right)\right), \tilde{\mathrm{r}}=a(t) r$ and $k$ denotes the spatial curvature and $k=0,1,-1$ correspond to open, flat, and closed universes respectively. The apparent horizon is located at

$$
\tilde{r}_{A}=\frac{1}{\sqrt{H^{2}+k / a^{2}}},
$$

where $H=\dot{a} / a$ is the Hubble parameter and over dot stands for the derivative with respect to cosmic time $t$. The apparent horizon has an associated entropy $(S)$ and Hawking temperature $(T)$ as

$$
S=\frac{A}{4 G}, T=\frac{1}{2 \pi \tilde{r}_{A}},
$$

where $A=n \Omega_{n} \tilde{\mathrm{r}}_{A}^{n-1}$ is the apparent horizon area and

$\Omega_{n}=\frac{\pi^{n / 2}}{\Gamma(n / 2+1)}$ being the volume of an d-dimensional unit sphere.

The Friedmann equations describe the dynamical evolution of the homogeneous and isotropic universe within the context of general relativity. Such equations can be derived by using Clausius relation to the apparent horizon of FRW universe, in which entropy is assumed to be a function of its horizon area.

Suppose the matter source in the FRW universe is a perfect fluid with stress-energy tensor

$$
T_{a b}=(p+\rho) u_{a} u_{b}+p g_{a b},
$$

where $p$ and $\rho$ are pressure and energy density respectively. Conservation law

$$
\begin{aligned}
& \nabla_{a} T^{a b}=0 \text { implies } \\
& \dot{\rho}+n H(\rho+p)=0 .
\end{aligned}
$$

Accordingly, we define the work density as ${ }^{45-48}$

$$
W=-\frac{1}{2} T^{a b} h_{a b},
$$

which results in

$$
W=\frac{1}{2}(\rho-p)
$$

The work density term is regarded as the work done by the change of the apparent horizon. The amount of energy crossing the apparent horizon during the time internal $d t$ is $^{13}$

$$
d E=A(\rho+p) H \mathrm{r}_{A} d t
$$

Now, applying the Clausius relation to the apparent horizon and assuming the entropy as a function of horizon area we can write

$$
A(\rho+p) H \tilde{r}_{A} d t=\frac{S^{\prime}(A)}{2 \pi \tilde{r}_{A}} d A .
$$

Making use of the relation $d \mathrm{r}_{A} / d t=-H\left(\dot{H}-k / a^{2}\right) \mathrm{r}_{A}^{3}$ in Eq. (2.9) after some simplifications one obtains ${ }^{45,46}$

$$
\left(\dot{H}-\frac{k}{a^{2}}\right) S^{\prime}(A)=-\frac{2 \pi}{n-1}(\rho+p),
$$

and noting the continuity equation (2.5) after some simple calculations we have

$$
\frac{2 \pi}{n} \rho=-\int S^{\prime}(A)\left(\frac{n \Omega_{n}}{A}\right)^{2 /(n-1)} \frac{d A}{A},
$$

where $S$ is assumed to be a function of horizon area $A$ and prime stands for derivative with respect to area.

As a simple example to show the application of Eqs.(2.10) and (2.11), making use of the entropy-area relation (2.3) in these equations, we have

$$
\begin{aligned}
\dot{H}-\frac{k}{a^{2}} & =-\frac{8 \pi G}{n-1}(\rho+p), \\
H^{2}+\frac{k}{a^{2}} & =\frac{16 \pi G}{n(n-1)} \rho .
\end{aligned}
$$

Equations (2.12) and (2.13) are the Friedmann equations in ( $\mathrm{n}+1)$ dimensional FRW universe obtained from the usual entropy-area relation. 
In the following sections, making use of the corrected functional form of the entropy stem from GUP and MDR analysis in Eqs.(2.10) and (2.11) we obtain the corrections to the Friedmann equations within both the GUP and the MDR respectively.

\section{The GUP corrections to the Friedmann equations}

To study the quantum gravity effects on the Friedmann equations, we employ the GUP. It is shown that usual uncertainty principle receives a modification at the microphysics regime. ${ }^{49-53}$

$$
\Delta x \geq \frac{h}{\Delta p}+\alpha L_{P}^{2} \frac{\Delta p}{h},
$$

where $L_{p}=\left(\frac{h G}{c^{3}}\right)^{1 /(n-1)}$, is the $(\mathrm{n}+1)$-dimensional Planck length with the order of $10^{-35} m$ for $n=3$. The term $\alpha L_{p}^{2} \frac{\Delta p}{h}$ in Eq.(3.1)

shows the gravitational effects on the usual uncertainty principle. Inverting Eq.(3.1) we obtain

$$
\frac{\delta x}{2 \alpha L_{P}^{2}}\left(1-\sqrt{1-\frac{4 \alpha L_{P}^{2}}{(\delta x)^{2}}}\right) \leq \frac{\Delta p}{h} \leq \frac{\delta x}{2 \alpha L_{P}^{2}}\left(1+\sqrt{1-\frac{4 \alpha L_{P}^{2}}{(\delta x)^{2}}}\right) .
$$

From Eq.(3.2), one can write

$$
\left(\frac{\Delta p}{h}\right)_{\min }=\frac{1}{\delta x}\left[\frac{(\delta x)^{2}}{2 \alpha L_{P}^{2}}\left(1-\sqrt{1-\frac{4 \alpha L_{P}^{2}}{(\delta x)^{2}}}\right)\right]=\frac{1}{\delta x} F_{(G U P)}\left((\delta x)^{2}\right),
$$

where

$$
F_{(G U P)}\left((\delta x)^{2}\right)=\frac{(\delta x)^{2}}{2 \alpha L_{P}^{2}}\left(1-\sqrt{1-\frac{4 \alpha L_{P}^{2}}{(\delta x)^{2}}}\right)
$$

characterizes the departure of GUP from the usual uncertainty principle.

Now we consider the impact of GUP on the entropy and Friedmann equations in the higher dimensional FRW universe. By considering the space-time having radiation or absorbtion at the horizon, one can identify the energy of the absorbed or radiated particles as the uncertainty of momentum, ${ }^{54}$ that is

$$
d E \simeq \delta p .
$$

The increase or decrease in the area of the black hole horizon can be written as

$$
d A=\frac{4 G}{T} d E \simeq \frac{4 G}{T} \frac{1}{\delta x} .
$$

When the gravitation is turned on Eq.(3.6) generalizes to

$$
d A_{(G U P)}=\frac{4 G}{T} d E \simeq \frac{4 G}{T} \frac{1}{\delta x} F_{(G U P)}\left((\delta x)^{2}\right)
$$

Combining Eqs.(3.6) and (3.7) we have

$$
d A_{(G U P)}=F_{(G U P)}\left((\delta x)^{2}\right) d A .
$$

By modelling the black hole as a cube of size $2 r_{A}$, the uncertainty in the position of a Hawking emitted particle is

$$
\delta x=2 \tilde{r}_{A}=2\left(\frac{A}{n \Omega_{n}}\right)^{\frac{1}{n-1}},
$$

from which $F_{(G U P)}$ can be rewritten as the function of $A$

$$
F_{(G U P)}(A)=\frac{2}{\alpha L_{P}^{2}}\left(\frac{A}{n \Omega_{n}}\right)^{\frac{2}{n-1}}\left[1-\sqrt{1-\alpha L_{P}^{2}\left(\frac{n \Omega_{n}}{A}\right)^{\frac{2}{n-1}}}\right] .
$$

Expanding (3.10) around $L_{P}=0$ gives,

$$
F_{(G U P)}(A)=1+\frac{\alpha L_{P}^{2}}{4}\left(\frac{n \Omega_{n}}{A}\right)^{\frac{2}{n-1}}+\frac{\left(\alpha L_{P}^{2}\right)^{2}}{8}\left(\frac{n \Omega_{n}}{A}\right)^{\frac{4}{n-1}}+\frac{5\left(\alpha L_{P}^{2}\right)^{3}}{64}\left(\frac{n \Omega_{n}}{A}\right)^{\frac{6}{n-1}}+\cdots
$$

and Eq.(3.8) can be rewritten as

$$
d A_{(G U P)}=F_{(G U P)}(A) d A .
$$

It must be noted that since $L_{P} \simeq 10^{-35} \mathrm{~m}$ the series expansion (3.11) will converge. This is why one can treat the GUP impacts perturbatively. ${ }^{55}$

Now by considering the impacts of GUP, Eqs.(2.9) and (2.10) can be written in the following form

$$
\begin{aligned}
& \left(\dot{H}-\frac{k}{a^{2}}\right) S_{(G U P)}^{\prime}(A)=-\frac{2 \pi}{n-1}(\rho+p), \\
& \frac{2 \pi}{n} \rho=-\int S_{(G U P)}^{\prime}(A)\left(\frac{n \Omega_{n}}{A}\right)^{2 /(n-1)} \frac{d A}{A},
\end{aligned}
$$

where $S_{(G U P)}(A)$ is defined as

$$
S_{(G U P)}(A)=\frac{A_{(G U P)}}{4 G},
$$

and considering Eq.(3.12), we can obtain

$$
S_{(G U P)}^{\prime}(A)=\frac{F_{(G U P)}(A)}{4 G} .
$$

Substituting Eqs.(3.11) and (3.16) in Eqs.(3.13) and (3.14), we can obtain the corrections to the Friedmann equations by considering the GUP, that is

$$
\begin{aligned}
& \left(\dot{H}-\frac{k}{a^{2}}\right)\left[1+\frac{\alpha L_{P}^{2}}{4}\left(\frac{n \Omega_{n}}{A}\right)^{\frac{2}{n-1}}+\frac{\left(\alpha L_{P}^{2}\right)^{2}}{8}\left(\frac{n \Omega_{n}}{A}\right)^{\frac{4}{n-1}}+\frac{5\left(\alpha L_{P}^{2}\right)^{3}}{64}\left(\frac{n \Omega_{n}}{A}\right)^{\frac{6}{n-1}}\right]=-\frac{8 \pi G}{n-1}(\rho+p), \\
& \left(H^{2}+\frac{k}{a^{2}}\right)\left[1+\frac{\alpha L_{P}^{2}}{8}\left(\frac{n \Omega_{n}}{A}\right)^{\frac{2}{n-1}}+\frac{\left(\alpha L_{P}^{2}\right)^{2}}{24}\left(\frac{n \Omega_{n}}{A}\right)^{\frac{4}{n-1}}+\frac{5\left(\alpha L_{P}^{2}\right)^{3}}{256}\left(\frac{n \Omega_{n}}{A}\right)^{\frac{6}{n-1}}\right]=\frac{16 \pi G}{n(n-1)} \rho,
\end{aligned}
$$


up to the sixth power of the Planck length. The terms proportional to powers of $\alpha L_{P}^{2}$ are the corrections to the Friedmann equations in $(\mathrm{n}+1)$-dimensional FRW universe. The correction terms are calculated based on the GUP.

It is well-known that corrected entropy-area relation depends on the dimension of the space- time. ${ }^{56-58}$ But Eqs.(3.17) and (3.18), the so-called generalized Friedmann equations, are independent on the dimension of the space-time and valid when $n$ is an odd or even number for $n \geq 3$. Also the generalized Friedmann equations are valid for any spacial curvature $k$.

By combining Eqs.(2.2) and (3.9) we arrived at the following relation

$$
\frac{1}{\tilde{r}_{A}}=\left(\frac{n \Omega_{n}}{A}\right)^{\frac{1}{n-1}}=\sqrt{H^{2}+\frac{k}{a^{2}}},
$$

in Eqs.(3.17) and (3.18), the generalized Friedmann equations can be rewritten as

$$
\begin{aligned}
\left(\dot{H}-\frac{k}{a^{2}}\right)[1+ & \frac{\alpha L_{P}^{2}}{4}\left(H^{2}+\frac{k}{a^{2}}\right)+\frac{\left(\alpha L_{P}^{2}\right)^{2}}{8}\left(H^{2}+\frac{k}{a^{2}}\right)^{2} \\
+ & \left.\frac{5\left(\alpha L_{P}^{2}\right)^{3}}{64}\left(H^{2}+\frac{k}{a^{2}}\right)^{3}\right]=-\frac{8 \pi G}{n-1}(\rho+p) \\
\left(H^{2}+\frac{k}{a^{2}}\right)[1+ & \frac{\alpha L_{P}^{2}}{8}\left(H^{2}+\frac{k}{a^{2}}\right)+\frac{\left(\alpha L_{P}^{2}\right)^{2}}{24}\left(H^{2}+\frac{k}{a^{2}}\right)^{2} \\
+ & \left.\frac{5\left(\alpha L_{P}^{2}\right)^{3}}{256}\left(H^{2}+\frac{k}{a^{2}}\right)^{3}\right]=\frac{16 \pi G}{n(n-1)} \rho
\end{aligned}
$$

Eqs.(3.20) and (3.21) are the quantum corrected Friedmann equations based on the GUP. They have the same form as those obtained in literatures which have been obtained from other alternative approaches..$^{41-43,45,46}$ Also, the results have the same structure as those obtained from purturbative quantum gravity.

It must be noted that the correction terms in Eqs.(3.20) and (3.21), as the basic equations of the quantum cosmology, are considerable if the scale factor $a$ is very small. It means that the corrections make sense only at the early stage of the universe where $a \rightarrow 0$. As the universe becomes larger, the correction terms become negligible and generalized Friedmann equations are reduced to the standard Friedmann equations in FRW universe. In other words, they do not affect the late time cosmology. The generalized Friedmann equations are expected to be useful to characterize the history of universe inflation and to resolve the big bang singularities. ${ }^{59}$

\section{The MDR corrections to the Friedmann equations}

In the study on loop quantum gravity and of models based on noncommutative geometry, there has been a strong interest in some candidate modifications of the energy-momentum dispersion relation. In this section, we introduce the extra dimensional version of MDR and investigate its impacts on the Friedmann equations in the higher dimensional FRW universe. Although the GUP, as an equivalence face of the MDR has been used extensively in the past, the extra dimensional MDR is new. ${ }^{39}$
It is interesting that the usual relation between energy and momentum that characterizes the special theory of relativity, $p^{2}=E^{2}-m^{2}$, may be modified in the Planck scale regime.

Anomalies in ultra high cosmic ray photons, and possibly Tev photons, may be explained by modification of the dispersion relation as $^{39,60-63}$

$$
\vec{p} \cdot \vec{p} \equiv p^{2}=f\left(E, m ; L_{P}\right)=E^{2}-\mu^{2}+\alpha_{1} L_{P}^{2} E^{4}+\alpha_{2} L_{P}^{4} E^{6}+\alpha_{3} L_{P}^{6} E^{8}+\mathcal{O}\left(L_{P}^{8} E^{10}\right),
$$

where $f$ is the function that gives the exact dispersion relation, and on the right-hand side we have assumed a Taylor-series expansion for

$E \ll \frac{1}{L_{P}}$. The coefficients $\alpha_{i}$ can take different values in different

quantum-gravity proposals. Note that $m$ is the rest energy of the particle and the mass parameter $\mu$ on the right-hand side is directly related to the rest energy, but $\mu \neq m$, if the $\alpha_{i}$ do not all vanish. Now differentiation of Eq.(4.1) and taking the inverse of the result gives

$$
\begin{array}{r}
d E=d p\left[1-\frac{3 \alpha_{1}}{2} L_{P}^{2} E^{2}-\left(\frac{5 \alpha_{2}}{2}-\frac{23 \alpha_{1}^{2}}{8}\right) L_{P}^{4} E^{4}\right. \\
\left.+\left(\frac{37}{4} \alpha_{1} \alpha_{2}-\frac{91}{16} \alpha_{1}^{3}-\frac{7}{2} \alpha_{3}\right) L_{P}^{6} E^{6}+\cdots\right]
\end{array} .
$$

Within quantum field theory, the relation between particle localization and its energy is given by

$$
E \geq \frac{1}{\delta x} \text {, where } \delta x \text { is particle position uncertainty. Now, it is }
$$

obvious that within MDR, this relation should be modified. To the first order, assuming $d E \simeq \delta E$, making use of the usual uncertainty $\mathrm{p}$ rinciple $\delta E \simeq \delta p \simeq \frac{1}{\delta x}$ we have

$$
\begin{aligned}
d E_{(M D R)} \simeq \frac{1}{\delta x} & {\left[1-\frac{3 \alpha_{1}}{2} \frac{L_{P}^{2}}{(\delta x)^{2}}-\left(\frac{5 \alpha_{2}}{2}-\frac{23 \alpha_{1}^{2}}{8}\right) \frac{L_{P}^{4}}{(\delta x)^{4}}\right.} \\
& \left.+\left(\frac{37}{4} \alpha_{1} \alpha_{2}-\frac{91}{16} \alpha_{1}^{3}-\frac{7}{2} \alpha_{3}\right) \frac{L_{P}^{6}}{(\delta x)^{6}}+\cdots\right]
\end{aligned}
$$

Noting (3.5), Eq.(4.3) can be rewritten as

$$
d E_{(M D R)}=\frac{1}{\delta x} F_{(M D R)}\left((\delta x)^{2}\right),
$$

and Eq.(3.12) must be replaced by

$$
d A_{(M D R)}=F_{(M D R)}(A) d A,
$$

where

$$
\begin{array}{r}
F_{(M D R)}(A)=1-\frac{3 \alpha_{1}}{8}\left(\frac{n \Omega_{n}}{A}\right)^{\frac{2}{n-1}} L_{P}^{2}-\left(\frac{5 \alpha_{2}}{2}-\frac{23 \alpha_{1}^{2}}{8}\right)\left(\frac{n \Omega_{n}}{A}\right)^{\frac{4}{n-1}} \frac{L_{P}^{4}}{16} \\
+\left(\frac{37}{4} \alpha_{1} \alpha_{2}-\frac{91}{16} \alpha_{1}^{3}-\frac{7}{2} \alpha_{3}\right)\left(\frac{n \Omega_{n}}{A}\right)^{\frac{6}{n-1}} \frac{L_{P}^{6}}{64}+\cdots
\end{array}
$$

Noting the value of the Planck length, one can ensure that this series expansion converges and impacts of MDR can be considered perturbatively. ${ }^{55}$ 
Making use of Eqs.(2.9) and (2.10) in the framework of MDR, we have

$$
\begin{aligned}
& \left(\dot{H}-\frac{k}{a^{2}}\right) S_{(M D R)}^{\prime}(A)=-\frac{2 \pi}{n-1}(\rho+p), \\
& \frac{2 \pi}{n} \rho=-\int S_{(M D R)}^{\prime}(A)\left(\frac{n \Omega_{n}}{A}\right)^{2 /(n-1)} \frac{d A}{A},
\end{aligned}
$$

where $S_{(M D R)}(A)$ is defined as

$$
S_{(M D R)}(A)=\frac{A_{(M D R)}}{4 G}
$$

and considering Eq.(3.12), we can obtain

$$
S_{(M D R)}^{\prime}(A)=\frac{F_{(M D R)}(A)}{4 G}
$$

Substituting Eqs.(4.6) and (4.10) in Eqs.(4.7) and (4.8), we can obtain the impacts of MDR to the Friedmann equations, that is

$$
\begin{aligned}
&\left(\dot{H}-\frac{k}{a^{2}}\right)[1-\frac{3 \alpha_{1} L_{P}^{2}}{8}\left(\frac{n \Omega_{n}}{A}\right)^{\frac{2}{n-1}}-\left(\frac{5 \alpha_{2}}{2}-\frac{23 \alpha_{1}^{2}}{8}\right)\left(\frac{n \Omega_{n}}{A}\right)^{\frac{4}{n-1}} \frac{L_{P}^{4}}{16} \\
&\left.+\left(\frac{37}{4} \alpha_{1} \alpha_{2}-\frac{91}{16} \alpha_{1}^{3}-\frac{7}{2} \alpha_{3}\right)\left(\frac{n \Omega_{n}}{A}\right)^{\frac{6}{n-1}} \frac{L_{P}^{6}}{64}\right]=-\frac{8 \pi G}{n-1}(\rho+p)
\end{aligned}
$$

$$
\begin{aligned}
&\left(H^{2}+\frac{k}{a^{2}}\right) {\left[1-\frac{3 \alpha_{1} L_{P}^{2}}{16}\left(\frac{n \Omega_{n}}{A}\right)^{\frac{2}{n-1}}-\left(\frac{5 \alpha_{2}}{2}-\frac{23 \alpha_{1}^{2}}{8}\right)\left(\frac{n \Omega_{n}}{A}\right)^{\frac{4}{n-1}} \frac{L_{P}^{4}}{48}\right.} \\
&\left.+\left(\frac{37}{4} \alpha_{1} \alpha_{2}-\frac{91}{16} \alpha_{1}^{3}-\frac{7}{2} \alpha_{3}\right)\left(\frac{n \Omega_{n}}{A}\right)^{\frac{6}{n-1}} \frac{L_{P}^{6}}{256}\right]=\frac{16 \pi G}{n(n-1)} \rho
\end{aligned}
$$

up to the sixth power of the Planck length. The terms proportional to powers of $L_{P}^{2}$ are the corrections to the Friedmann equations in $(\mathrm{n}+1)$-dimensional FRW universe. The correction terms are calculated based on the MDR.

From the literature ${ }^{5-58}$ the corrected entropy-area relation stem from MDR, depends on the dimension of the space-time. But Eqs. (4.11) and (4.12), the so-called modified Friedmann equations, are valid for all values of $n \geq 3$ and all values of spacial curvature $k$.

Substituting Eq.(3.19) in Eqs.(4.11) and (4.12) leads to

$$
\begin{aligned}
&\left(\dot{H}-\frac{k}{a^{2}}\right)[ 1-\frac{3 \alpha_{1} L_{P}^{2}}{8}\left(H^{2}+\frac{k}{a^{2}}\right)-\left(\frac{5 \alpha_{2}}{2}-\frac{23 \alpha_{1}^{2}}{8}\right)\left(H^{2}+\frac{k}{a^{2}}\right)^{2} \frac{L_{P}^{4}}{16} \\
&\left.+\left(\frac{37}{4} \alpha_{1} \alpha_{2}-\frac{91}{16} \alpha_{1}^{3}-\frac{7}{2} \alpha_{3}\right)\left(H^{2}+\frac{k}{a^{2}}\right)^{3} \frac{L_{P}^{6}}{64}\right]=-\frac{8 \pi G}{n-1}(\rho+p)
\end{aligned}
$$

$$
\begin{aligned}
&\left(H^{2}+\frac{k}{a^{2}}\right) {\left[1-\frac{3 \alpha_{1} L_{P}^{2}}{16}\left(H^{2}+\frac{k}{a^{2}}\right)-\left(\frac{5 \alpha_{2}}{2}-\frac{23 \alpha_{1}^{2}}{8}\right)\left(H^{2}+\frac{k}{a^{2}}\right)^{2} \frac{L_{P}^{4}}{48}\right.} \\
&\left.+\left(\frac{37}{4} \alpha_{1} \alpha_{2}-\frac{91}{16} \alpha_{1}^{3}-\frac{7}{2} \alpha_{3}\right)\left(H^{2}+\frac{k}{a^{2}}\right)^{3} \frac{L_{P}^{6}}{256}\right]=\frac{16 \pi G}{n(n-1)} \rho
\end{aligned}
$$

Eqs.(4.13) and (4.14) show the explicit form of the quantum corrected Friedmann equations arising from MDR. They are identical in form to those recorded by several other authors obtained from alternative approaches. ${ }^{41-46}$

Just like the case of GUP corrections, the correction terms make sense only at the early stage of the universe where the physical scales as well as the universe scale factor are in the same order as Planck scale. They are expected to have some consequences on inflation and early time cosmology. ${ }^{59}$ As the universe becomes larger, the correction terms become negligible and the so-called modified Friedmann equations are reduced to the standard Friedmann equations in FRW universe. It means that the correction terms seem to have no influence on the late cosmology.

\section{Comparison of the results}

Since GUP and MDR are different manifestations of the same physical concept (existence of a minimal length scale of the same order of Planck length) we expect the results of applications of these two alternative approaches to the physical systems to be identical.

In the two previous sections we examined the GUP and MDR separately and obtained the corrections to the entropy as well as the Friedmann equations in $(\mathrm{n}+1)$-dimensional FRW universe. Now it is evident to expect the results of these two alternative approaches to be consistent. The assumption behind this expectation is that GUP and MDR are two faces of an underlying quantum gravitational proposal.

Through direct comparison of the results obtained we found that by using the suitable choice of the coefficients, that is

$$
\alpha_{1}=-\frac{2}{3} \alpha, \text { and } \alpha_{2}=-\frac{13}{45} \alpha^{2}, \text { and } \alpha_{3}=-\frac{46}{105} \alpha^{3},
$$

in Eqs.(3.17), (3.18), (4.11) and (4.12), one can show that the generalized and modified Friedmann equations obtained from GUP and MDR analysis respectively are identical. The results are compatible with that of ref. ${ }^{56-58}$ As a mathematical result it seems that GUP and MDR approaches lead to the same corrections to the Friedmann equations, if the coefficients are fixed properly.

\section{Conclusion}

Recent studies in perturbative string theory and loop quantum gravity predict the existence of a fundamental measurable length which is of the order of Planck length. The essence of this fundamental length can be captured by generalizing usual uncertainty principle known as GUP or by modifying the usual energy momentum relation known as MDR.

In this work, the corrections to the Friedmann equations in the higher dimensional FRW universe are investigated in the presence of 1) the generalized uncertainty principle and 2) the modified dispersion relation. We showed that the Friedmann equations receive 
some new corrections at the Planck scale as Eqs.(3.17) and (3.18) (or Eqs.(3.20) and (3.21)) within the framework of GUP and Eqs. 4.114.14 within the framework of MDR. The corrections are worked out up to the sixth power of the Planck length. The quantum corrected Friedmann equations we just obtained, (Eqs.(3.20) and (3.21)) or (Eqs.(4.13) and (4.14)) are consistent with numerous other studies that have delved into the subject of this paper. Also, they are identical in form to those arisen from perturbative quantum gravity. It must be emphasized that the quantum corrected terms are considerable only in the early time cosmology where the scale factor $a$ is very small and the physical scales are in the order comparable with the Planck scale. As the universe becomes larger the quantum corrected terms can be neglected and the quantum gravity corrected Friedmann equations are reduced to the standard form in the FRW universe. It seems that the quantum gravity corrected Friedmann equations do not influence the late time cosmology. Therefore, it is expected that they have some new consequences on characterizing the primordial inflation and resolving the big bang singularities.

It is significant that one can obtain the GUP or MDR by starting from modified momentum operator. From this point of view, GUP and MDR are two faces of the same physical concept (existence of a minimal length scale of the same order of Planck length) and it is evident to expect the results of these two approaches to be consistent. Through the comparison of the corrected Friedmann equations, obtained from these two alternative approaches, we found that a suitable choice of the expansion coefficients in the MDR (Eq. 5.1) leads to the same results in both approaches.

\section{Acknowledgments}

None

\section{Conflicts of interest}

Authors declare there is no conflict of interest.

\section{References}

1. MH Dehghani, SH Hendi. Wormhole solutions in Gauss-Bonnet-BornInfeld gravity. Gen Rel Grav. 2009;41(8):1853-1863.

2. R Emparan, HS Reall. Black Holes in Higher Dimensions. Living Rev Relativity. 2008;11:6.

3. T Jacobson. Thermodynamics of Spacetime: The Einstein Equation of State. Phys Rev Lett. 1995;75(7):1260.

4. SW Hawking. Black hole explosions? Nature. 1974;248:30-31.

5. SW Hawking. Particle creation by black holes. Commun Math Phys 1975;43:199-220.

6. JD Bekenstein. Black Holes and Entropy. Phys Rev D. 1973;7(8):2333.

7. C Eling, R Guedens, T Jacobson. Nonequilibrium Thermodynamics of Spacetime. Phys Rev Lett. 2006;96:121301.

8. M Akbar, RG Cai. Friedmann Equations of FRW Universe in Scalar-tenso Gravity, $\mathrm{f}(\mathrm{R})$ Gravity and First Law of Thermodynamics. Phys Lett B. 2006;635(1):7-10.

9. M Akbar, RG Cai. Thermodynamic behavior of field equations for $f(\mathrm{R})$ gravity. Phys Lett B. 2007;648(2-3):243.

10. T Padmanabhan. Classical and quantum thermodynamics of horizons in spherically symmetric spacetimes. Class Quantum Grav. 2002;19(21):5387.
11. T Padmanabhan. Gravity and the Thermodynamics of Horizons. Phys Rept. 2005;406(2):49-125

12. A Paranjape, S Sarkar, T Padmanabhan. Thermodynamic route to field equations in Lanczos-Lovelock gravity. Phys Rev D. 2006;74:104015.

13. RG Cai, LM Cao, N Ohta. Friedmann equations from entropic force. Phys Rev D. 2010;81:061501.

14. A Sheykhi. Thermodynamics of apparent horizon and modified Friedmann equations. Eur Phys J C. 2010;69(1-2):265-269.

15. A Sheykhi, SH Hendi. Power-law entropic corrections to Newton's law and Friedmann equations. Phys Rev D. 2011;84:044023.

16. R Gambini, J Pullin. Nonstandard optics from quantum space-time. Phys Rev D. 1999;59:124021.

17. J Alfaro, HA. Morales Tecotl, LF Urrutia. Quantum Gravity Corrections to Neutrino Propagation. Phys Rev Lett I. 2000;84:2318.

18. J Lukierski, H Ruegg, WJ Zakrzewski. Classical and Quantum Mechanics of Free א-Relativistic Systems. Ann Phys. 1995;243:90-116.

19. G Veneziano. A Stringy Nature Needs Just Two Constants. Euro Phys Lett. 1986;2(3):199.

20. DJ Gross, PF Mende. String theory beyond the Planck scale. Nucl Phys B. 1988;303(3):407-454.

21. D Amati, M Ciafaloni, G Veneziano. Can spacetime be probed below the string size. Phys Lett B. 1989;216(1-2):41.

22. K Konishi, G Paffuti, P Provero. Minimum physical length and the generalized uncertainty principle in string theory. Phys Lett B. 1990;234(3):276-284.

23. K Nouicer. Quantum-corrected black hole thermodynamics to all orders in the Planck length. Phys Lett B. 2007;646(2-3):63-71.

24. LXiang. Dispersion relation, black hole thermodynamics and generalization of uncertainty principle. Phys Lett B. 2006;638(5-6):519-522.

25. R Adler, P Chen, D Santiago. The Generalized Uncertainty Principle and Black Hole Remnants. Gen Rel Grav. 2001;33(12):2101-2108.

26. X Han, H Li, Y Ling. Modified dispersion relations and (A)dS Schwarzschild black holes. Phys Lett B. 2008;666(2):121-124.

27. YW Kim, YJ Park. Entropy of the Schwarzschild black hole to all orders in the Planck length. Phys Lett B. 2007;655(3-4):172-177.

28. D Amati, M Ciafaloni, G Veneziano. Superstring collisions at planckian energies. Phys Lett. 1987;81-88.

29. Z Ren, Z Sheng Li. Generalized uncertainty principle and black hole entropy. Phys Lett B. 2006;641(2):208-211.

30. M Maggior. Quantum groups, gravity, and the generalized uncertainty principle. Phys Rev D. 1994;49:5182.

31. MR Setare. Corrections to the Cardy-Verlinde formula from the generalized uncertainty principle. Phys Rev D. 2004;70:087501.

32. MR Setare. The Generalized Uncertainty Principle and Corrections to the Cardy-Verlinde Formula in SAdS 5 Black Holes. Int J Mod Phys A. 2006;21(6):1325.

33. AS Sephiedgar, HR Sepangi. Corrections to the Cardy-Verlinde formula from the modified dispersion relation in extra dimensions. Phys Lett B. 2012;706(4-5):431-435

34. T Zhu, JR MF Li. Influence of generalized and extended uncertainty principle on thermodynamics of FRW universe. Phys Lett B. 2009;674(3):204. 
35. WG Unruh. Sonic analogue of black holes and the effects of high frequencies on black hole evaporation. Phys Rev D. 1955;51:2827.

36. D Colladay, VA Kostelecky. Lorentz-violating extension of the standard model. Phys Rev D. 1998;58:116002.

37. SR Coleman, SL Glashow. High-energy tests of Lorentz invariance. Phys Rev D. 1999;59:116008.

38. RC Myers, M Pospelov. Ultraviolet Modifications of Dispersion Relations in Effective Field Theory. Phys Rev Lett. 2003;90:211601.

39. AS Sephiedgar, K Nozari, HR Sepangi. Modified dispersion relations in extra dimensions. Phys Lett B. 2010;696(1-2):119-123.

40. Liu B, Dai YH, Hu XR, et al. The Friedmann equation in modified entropyarea relation from entropy force. Mod Phys Lett. 2011;26(7):489-500.

41. A Sheykhi. Thermodynamics of apparent horizon and modified Friedmann equations. Eur Phys J C. 2010;69(1-2):265-269.

42. A Sheykhi. Entropic corrections to Friedmann equations. Phys Rev. 2010;81:104011.

43. A Sheykhi, $Z$ Teimoori. Modified Friedmann equations from Debye entropic gravity. Gen Rel Grav. 2012;44:1129-1149.

44. A Sheykhi, SH Hendi. Power-law entropic corrections to Newton's law and Friedmann equations. Phys Rev D. 2011;84:044023.

45. RG Cai, LM Cao, YP Hu. Corrected entropy-area relation and modified Friedmann equations. J High Energy Phys. 2008;08:090.

46. RG Cai, SP Kim. First law of thermodynamics and Friedmann equations of Friedmann-Robertson-Walker universe. J High Energy phys. 2005;02:050.

47. SA Hayward, S Mukohyana, MC Ashworth. Dynamic black-hole entropy. Phys Lett A. 1999;256:347.

48. SA Hayward. Unified first law of black-hole dynamics and relativistic thermodynamics. Class Quant Grav. 1998;15:3147.

49. SHossenfelder, MBleicher, H Stöcker et al. Probing the minimal length scale by precision tests of the muon $g-2$. Phys Lett B. 2004;584(1-2):109-113.
50. S Hossenfelder. Interpretation of quantum field theories with a minimal length scale. Phys Rev D. 2006;73:105013.

51. A Kempf, G Managano. Minimal length uncertainty relation and ultraviolet regularization. Phys Rev D. 1997;55:7909.

52. M Dehghani, A Farmany. Higher dimensional black hole radiation and a generalized uncertainty principle. Phys Lett B. 2009;675(5):460-462.

53. M Dehghani, A Farmany. GUP and higher dimensional ReissnerNordström black hole radiation. Braz J Phys. 2009;39(3):570-573.

54. MI Park. The generalized uncertainty principle in (A)dS space and the modification of Hawking temperature from the minimal length. Phys Lett B. 2008;659(3):698-702.

55. K Nozari, AS Sefiedgar. Comparison of approaches to quantum correction of black hole thermodynamics. Phys Lett B. 2006;635(2-3):156-160.

56. M Dehghani. Corrections to the Hawking tunneling radiation in extra dimensions. Phys Lett B. 2015;749:125-129.

57. M Dehghani. The Cardy-Verlinde formula in the presence of GUP and MDR. Astrophys Space Sci. 2016;361:148.

58. M Dehghani. Corrections to the Cardy-Verlinde formula at the Planck scale. New Astronomy. 2017;51:37-42.

59. Sergio del Campo. Approach to exact inflation in modified Friedmann equation. J Cosmol Astropart Phys. 2012:005.

60. G Amelino Camelia, J Ellis, NE Mavromatos, et al. Tests of quantum gravity from observations of $\gamma$-ray bursts. Nature. 1998;393:763-765.

61. R Aloisio, P Blasi, PL Ghia, et al. Probing the structure of space-time with cosmic rays. Phys Rev D. 2000;62:053010.

62. G Amelino Camelia, T Piran. Planck-scale deformation of Lorentz symmetry as a solution to the ultrahigh energy cosmic ray and the TeVphoton paradoxes. Phys Rev D. 2001;64:036005.

63. T Jacobson, S Liberati, D Mattingly. TeV astrophysics constraints on Planck scale Lorentz violation. Phys Rev D. 2002;66:081302. 